\title{
Self-Monitoring, Self-Healing Biomorphic Sensor Technology
}

\author{
Andrew Richardson \\ enablingMNT UK Ltd. \\ Dept. Engineering \\ Lancaster University \\ Lancaster, UK \\ A.Richardson@Lancaster.ac.uk
}

\author{
David Cheneler \\ Dept. Engineering \\ Lancaster University \\ Lancaster, UK \\ D.Cheneler@lancaster.ac.uk
}

\begin{abstract}
The deployment of autonomous sensors within electronic systems for both existing and emerging markets requires an increase in the reliability, security and dependability of the associated data generated. The availability of intelligent sensors that can self-adapt and ultimately self-heal would be a key step towards this objective. This paper presents ideas associated with the utilisation of sensor self-test principles and software algorithms able to generate sensor prognostics and drive adaptation, compensation and self-healing functions. Major initiatives supported both within Europe and further afield to migrate processing power to the "Edge", deploy $5 \mathrm{G}$ technologies and integrate Artificial Intelligence across the system hierarchy provide technological platforms to deliver many of these concepts. An example associated with simple printed electrodes targeting corrosion detection and potentially the detection of hydrogen is presented in the context of a step towards full biomorphic capability.
\end{abstract}

Keywords-self-test, self-monitoring, self-healing, design for testability, sensors.

\section{INTRODUCTION}

Sensing technologies that can both monitor their own condition and adapt to degradation and changing operational conditions have immediate applications in critical systems, harsh environments and real-time control across the majority of commercial sectors that utilise sensors. This paper proposes new design principles to deliver "biomorphism" or on-line fault detection and correction based on bio-inspired principles within sensor technologies through the integration of edge-based intelligence. In the future, combining these principles with self-healing materials promises to deliver full self-healing sensor solutions.

Prior art includes a number of designs for adaptable electronics together with methods for implementing on-line test and dynamic reconfiguration. Sensing layers, in particular MEMS based solutions, have been engineered with self-test and condition monitoring functions that utilise crosssensitivities between devices to drive compensation and also generate prognostics [1]. At the packaging level, laminate and embedded structures have been proposed that are able to monitor strain and temperature and morph the encapsulation into a communication structure, leading to the concept of functionalised packaging [2] [3]. All of these solutions have, however, been proposed for a specific active technology layer and hence do not represent complete systems solutions. This makes usability within a real intelligent sensor difficult as in most cases the physics of failure and environmental stress patterns associated with a real device do not necessarily impact all layers of the system. This leads to incomplete coverage of potential faults if only a subset of layers has a prognostic capability.

\section{Methodology}

Our approach to the realisation of "biomorphism" complements the materials engineering advances that have made possible the deployment of intelligent sensors in high temperature, vibration, and ionising environments possible. The concept is based on the premise that zero failure during system operation is unachievable through materials and assembly engineering alone. Our approach involves the integration of self-healing and self-adapting technology across the functional layers of autonomous sensor systems (electronics, sensing and packaging). This opens the possibility of realising products that emulate the resilience of living entities. The ability to "morph" i.e. to undergo a gradual process of transformation of the system in response to environment conditions and degradation using nature-inspired principles gives rise to the approach and promises to deliver a major paradigm shift in the electronics system community.

\section{TOOLBOX}

There are a number of methods to generate localised corrective feedback from sensors, in response to degradation in materials, geometrical structure or the operational environment. Some require edge-based computing resource and memory, others a communication infrastructure, a centralised processing engine and dedicated hardware.

"Stimulate \& Feel" methods require the use an electrical stimulus to excite the sensor and electrical reliability indictors sensitive to parameters related to the integrity of the sensor. The "bias superposition" [4] concept is a good example and a valuable tool but is limited to active devices and requires dedicated electrical stimuli superimposed onto the normal voltage or current bias. This method can be used on-line provided that the stimuli is outside of the operational bandwidth of the sensor. In the case where the application of this additional test stimuli couples into the normal operation response, degrading signal to noise ratio and / or dynamic range, DSP methods based around a digital stimuli can be used to improve the differentiation between normal and test response [5]. The strength of this method is its applicability to a range of sensing structures, and flexibility associated with implementation that ranges from simple sinusoidal stimuli and filtering through to more complex digital solutions. Its disadvantages include the necessity to add functionality resulting in an increase in the complexity of the electronics to provide the stimulus and the monitor that can also impact power consumption of the device. Furthermore, its use only 
provides a measure of the structural integrity of the sensor. It has however been shown that provided correlation can be established between the monitor output and the impact of associated degradation on the sensor response, the method could be combined with localised algorithms to generate gain and offset feedback to "correct" the sensor response and provide a degree of fault tolerance and self-adaptation.

Probe and Compare strategies include the use of crosssensitivities, normally eliminated within well designed systems to deliver validation data for specific primary sensor outputs. There is potential to use package loaded structures or co-located sensors, engineered with cross-sensitivities to parameters that need to be monitored. Here we can build on prognostic strategies that have been proposed in [1] that have significant potential in multi-functional biomorphic devices. In this particular example, a dual mode sensor has been realised that uses a single diaphragm, suitable patterned to measure both pressure and humidity. In array based applications that feature replicated dual mode devices, it is feasible to utilise the primary response to measure a key physical parameter and the secondary response as a check on a primary response from an adjacent device that is configured to measure the second physical parameter. The approach opens the potential for reconfiguration based self-repair where degrading devices can effectively be detected and switched out of the array.

"Replicate, Rotate and Compare" functions build on the dual mode sensing concept but require computing resource ideally at the edge. In this case, data sourced from "Stimulate and Feel" or "Probe and Compare" functions are used to either reconfigure a specific sensor or reconfigure an array of sensors. A fault tolerant bridge based sensor was proposed in [6] that utilised half bridges that could be rotated under digital control. The electrical position of theses bridges in the transducer structure was shown to result in minimal effects on the key sensor parameters but if one of these bridges featured material degradation, the functional response would differ from position to position. This method requires single or array based transducers to be designed using replicated physical or electronic structures. The switching functions are also critical especially where impedance and parasitics are injected into the signal path.

"Probe and Predict" technology utilises reliability indicators that are normally electrical parameters such as a voltage, current or frequency, sensitive to material or geometrical degradation together with prognostic algorithms. An example here is the use of self-oscillation [7] to drive prognostics associated with electrode degradation [8]. Here Linear Discriminant Analysis has been deployed through a low-power algorithm to provide a prognostic capability compatible with the limitations of edge based computing. The integrity of sensing devices, based on the monitoring and processing of Reliability Indicators across arrays of either identical sensing devices with a single sensing mode or arrays with programmable devices and multiple sensing modes can be also be achieved through the use adaptive filters. The challenge here will be to develop a low power usable algorithm that can implement strategies similar to [1] where validation data for each primary output is available and in cases where validation does not exist, an ability to statistically prognose device behaviour from data across the array. Distributed versions of the Least Squares Algorithm has potential with the use of special and temporal data.
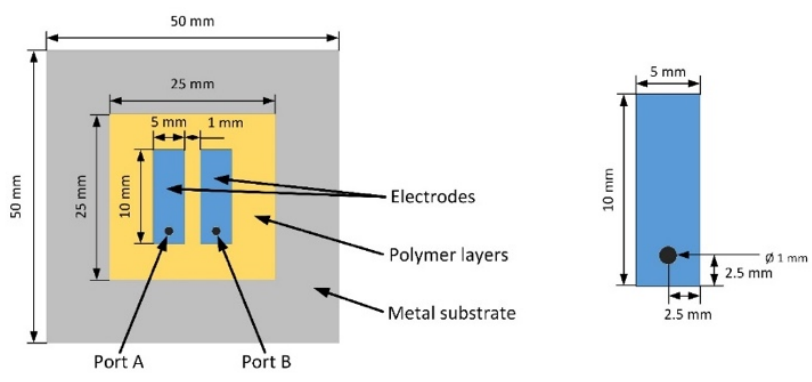

Fig. 1: Top view of sensor geometry. (Left) Complete layout. (Right) Close-up of electrode. NOT TO SCALE. Dimensions scaled for clarity.
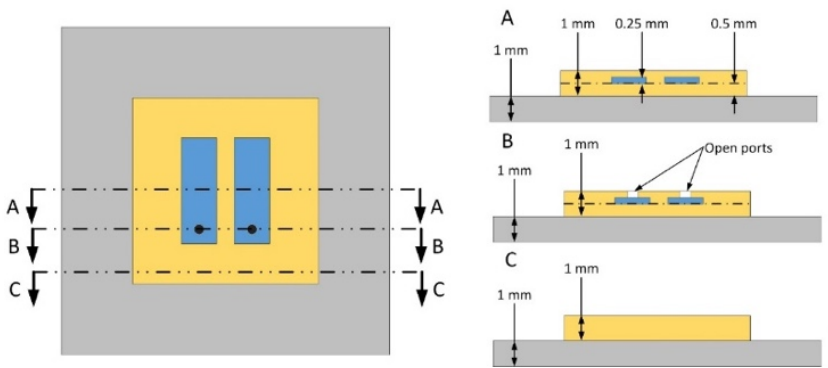

Fig. 2: Cross-sectional view of sensor geometry.

\section{CASE STUDY}

Corrosion sensing is critical to many safety critical and high reliability applications. Furthermore, in applications where access is difficult or costly, lifetime and validation of the condition of the sensor or sensors is of upmost importance. A new corrosion sensor design based on two isolated electrodes is shown in Fig. $1 \& 2$. The sensing methods used include Electrochemical Impedance Spectroscopy (EIS) [9] and Electrochemical Noise Measurements (ENM) [10]. In order to predict the efficacy of these techniques, electromagnetic simulations have been carried out to determine an equivalent network or circuit of the system.

Kapton is used as the packaging material. The electrodes are printed using a polymer solver paste ESL 1120 that is a silver filled, resin compound that cures at $150^{\circ} \mathrm{C}$ for printing conductors on ridged and flexible substrates. Resistivity, as measured on a $100 \mathrm{~mm} \times 10 \mathrm{~mm}$ conductor track is $20 \mathrm{~m} \Omega / \mathrm{sq}$. The target substrate material where corrosion needs to be detected is stainless steel type 1.307 also known as grade 304L. This is the low carbon version of 304 which has nominal composition of type 304 being $18 \%$ chromium and $8 \%$ nickel and is austenitic. The alloy's lower carbon content minimises chromium carbide precipitation due to welding and its susceptibility to intergranular corrosion and does not require post-weld annealing, as compared to 304, which does. Electrical Resistivity is $0.072 \mathrm{e}-6 \Omega \mathrm{m}$.

The boundary conditions applied within the simulation models are:

- Voltage applied to Port A

○ $10^{-4}$ to $10^{6} \mathrm{~Hz}$

○ $\pm 100 \mathrm{mV}$

- Port B is ground

- All other surfaces are isolated. 


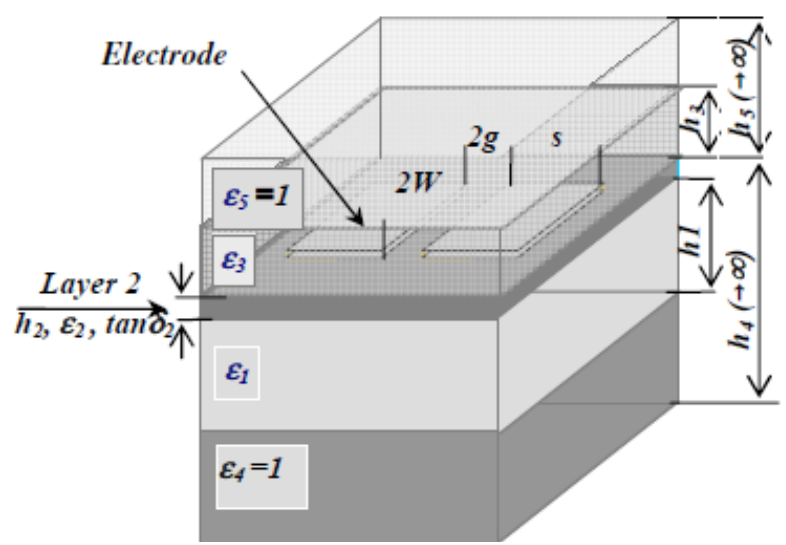

Fig. 3: Schematic of multilayer coplanar electrodes (from [13]).

The impedance sensors are formed by two coplanar conducting electrodes sandwiched between dielectric layers on top of a lossy substrate, i.e. the stainless steel. As such, the impedance of the sensor can be approximated using analytical expressions derived from conformal mapping techniques which take into account the finite dimensions of the electrodes and the various layers and fringing fields [11] [12] [13]. Here we will use the conventions defined in [13] and depicted in Fig. 3.

In the real application, layer 1 is the stainless-steel layer under investigation, layers 2 and 3 are the dielectric layers insulating the sensor and layers 4 and 5 are air of infinite height. From [13], the capacitance is:

$$
C=2 \varepsilon_{e f f} \varepsilon_{0} \frac{K\left(k_{a}^{\prime}\right)}{K\left(k_{a}\right)} s K\left(k_{0}^{\prime}\right)
$$

where $\varepsilon_{0}$ is the permittivity of free space and $s$ is the width of the electrodes. $K$ is the compete elliptic integral of the first kind with modulus, $m$ (see [13] for a full description). Assuming the following dimensions: $g=0.5 \mathrm{~mm}, W=25$ $\mathrm{mm}, \mathrm{s}=5 \mathrm{~mm}, h_{1}=1 \mathrm{~mm}, h_{2}=0.5 \mathrm{~mm}$ and $h_{3}=0.5 \mathrm{~mm}$ and the following properties: $\varepsilon_{1}=1.1, \sigma_{1}=1.4 \times 10^{6} \mathrm{~S} / \mathrm{m}, \varepsilon_{2}=$ $3.1, \sigma_{2}=1 \times 10^{-17} \mathrm{~S} / \mathrm{m}$, and $\varepsilon_{3}=3.1, \sigma_{3}=1 \times 10^{-17} \mathrm{~S} / \mathrm{m}$, the capacitance and conductance for the system as a function of $h_{1}$ (equivalent to material loss due to corrosion) results in the values listed in Table 1.

\begin{tabular}{|l|l|l|l|l|}
\hline & $\begin{array}{l}\text { Conductance } \\
(\mathrm{S})\end{array}$ & $\begin{array}{l}\text { Change } \\
(\%)\end{array}$ & $\begin{array}{l}\text { Capacitance } \\
(\mathrm{fF})\end{array}$ & $\begin{array}{l}\text { Change } \\
(\%)\end{array}$ \\
\hline $\begin{array}{l}\text { Full } \\
\text { thickness }\end{array}$ & 16437.68 & 0 & 2021.27 & 0 \\
\hline $10 \%$ loss & 13677.02 & 16.79 & 2199.53 & 0.08 \\
\hline $20 \%$ loss & 10684.80 & 34.99 & 2197.63 & 0.17 \\
\hline
\end{tabular}

Table 1: Conductance and capacitance of the system as function of reduction of thickness of layer 1

The frequency response of the system is shown in Fig. 4 $\& 5$. The impedance is dominated by the capacitance of the dielectric layers, as expected. The substrate of interest is naturally very conductive and adds a negligible amount to the overall capacitance. As such this does not change significantly with corrosion. The conductance does change with material loss due to corrosion by a relatively significant amount.

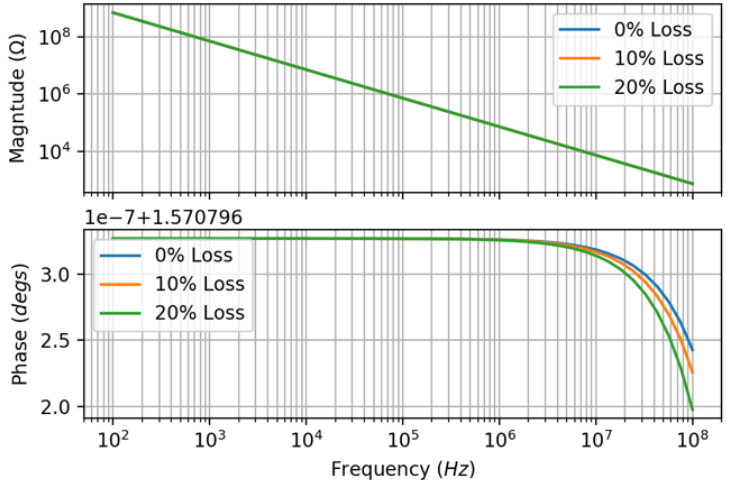

Fig. 4: Magnitude (top) and phase (bottom) of impedance of system as function of frequency
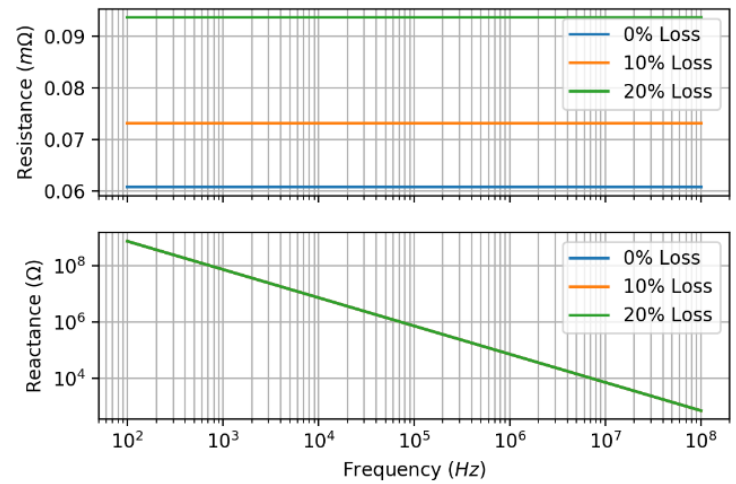

Figure 5: Resistance (top) and capacitance (bottom) of system as function of frequency

The above analysis assumes the sensor itself does not degrade. However, we can use the same analysis to show that the sensor can monitor its own level of degradation. The above analysis makes it clear that it is the conductance that changes as a function of substrate corrosion and that in the case of no degradation of the sensor, capacitance changes are negligible, these being a function of the sensors dielectric layers. This means that by monitoring the capacitance, we can detect degradation in these dielectric layers. If we assume that as we lose $10 \%$ of the substrate due to corrosion, that we also lose say $5 \%$ of the thickness of the dielectric layers, the change in capacitance is more than an order of magnitude larger than the change in capacitance due to the material loss in the substrate alone (see Table 2).

\begin{tabular}{|l|l|l|l|l|l|}
\hline Dielectric & Substrate & $\begin{array}{l}\text { Conductance } \\
(\mathrm{S})\end{array}$ & $\begin{array}{l}\text { Change } \\
(\%)\end{array}$ & $\begin{array}{l}\text { Capacitance } \\
(\mathrm{fF})\end{array}$ & $\begin{array}{l}\text { Change } \\
(\%)\end{array}$ \\
\hline $\begin{array}{l}\text { Full } \\
\text { thickness }\end{array}$ & $\begin{array}{l}\text { Full } \\
\text { thickness }\end{array}$ & 16437.68 & 0 & 2201.27 & 0 \\
\hline $5 \%$ loss & $10 \%$ loss & 13677.02 & 16.79 & 2178.38 & 1.04 \\
\hline $10 \%$ loss & $20 \%$ loss & 10684.80 & 34.99 & 2153.94 & 2.15 \\
\hline
\end{tabular}

Table 2: Conductance and capacitance of the system as function of reduction of thickness of the substrate and the dielectric layers.

\section{BIOMORPHIC STRUCTURE}

Given the simplicity of the sensor, arrays fabricated on sheets that can be bonded to the target substrate provides both redundancy and the opportunity for differential measurement addressing the need for high resolution associated with the 
detection of material loss. The cell in Figure 6 delivers a solution based around "Stimulate and Feel" that utilises an OBIST structure to deliver a frequency change as a function of both degradation of the target substrate due to corrosion and the physical structure of the sensor. Sensor degradation will be frequency dependant, corrosion effects are not. Hence, by taking differential measurements across the array at multiple frequencies, corrosion can be detected and differentiated from sensor degradation effects. Given that any cell can be set as master with the response of each cell in the array compared to this master response, failing cells can be switched out of the structures and degrading cells corrected by applying a compensation bias (frequency or amplitude) back into the OBIST function. The Tx and Rx function can be wired or wireless.
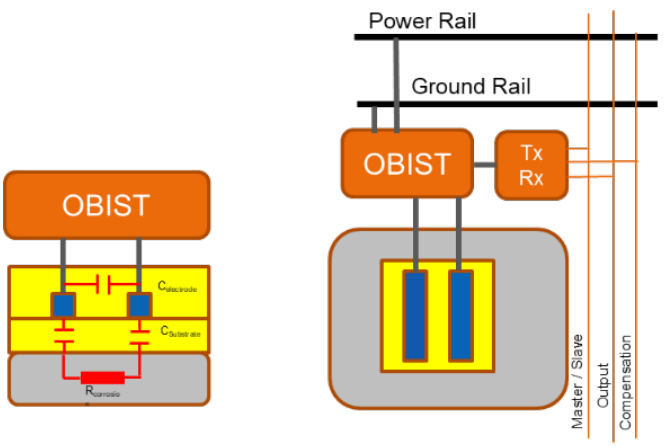

Fig. 6: Single corrosion cell with biomorphic functions

The array requires a single edge-based controller to configure the cells and deliver both readings and condition to a central controller or cloud based surveillance system.

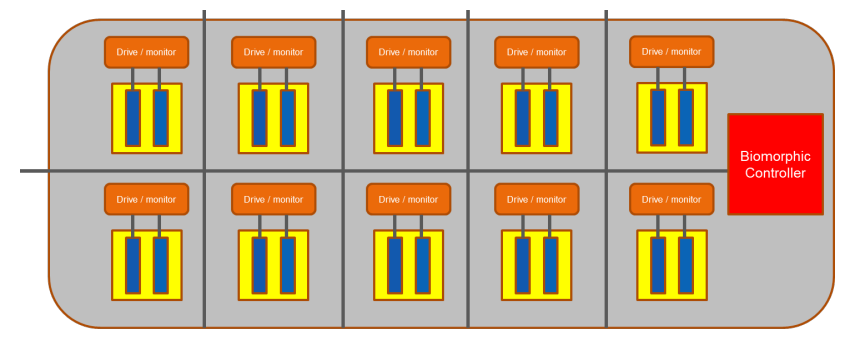

Fig. 7 Array based implementation

\section{CONCLUSIONS}

The emergence of low power edge based processing opens the opportunity to build prognostics and self-correcting functions into autonomous sensors. A toolbox of stimulus and response analysis functions could well open a pathway for designers to deliver the required functionality against degradation models of their devices. Prognostic functions that predict failure from trends in Reliability Indicators or from correlation between different parameters are likely to become more practical with the emergence of edge-based computing. In the future, selfhealing materials could provide a way for prognostic functions to activate healing processes for interconnect, transducer and package structures.

\section{FUTURE WORK}

Work will focus on the fabrication of the corrosion sensor and the implementation of the OBIST structure within a benchtop prototype. Design enhancements to support the detection of hydrogen will also be explored. Two modifications would be required. First the electrode array would be interdigitated rather than the linearly spaced implementation shown in Fig.
7. Additionally, a layer of palladium-based metal hydride would be deposited on top of the electrodes and a package option to allow exposure from the top implemented. The swelling of the sensitive layer would be detected by a change in capacitance between adjacent electrodes using the OBIST architecture presented.

\section{VIII.ACKNOWLEDGMENTS}

The authors would like to thank Frank Allison from FIS360 for supporting this work and Sellafield Ltd through their "Game Changers" program for funding the development of the corrosion sensor.

\section{REFERENCES}

[1] A. Richardson, "Use of Self-Calibration Data for Multifunctional MEMS Sensor Prognostics," IEEE Journal of Micromechanical Systems, vol. 25, no. 4, pp. 761-769, 2016.

[2] M. Hassan and et al, "Combining organic and printed electronics in Hybrid System in Foil (HySiF) based smart skin for robotic applications," in European Microelectronics Packaging Conference (EMPC), Friedrichshafen, 2015.

[3] B. J. Blaiszik, S. L. B. Kramer, S. C. Olugebefola, J. S. Moore, N. R. Sottos and S. R. A. White, "Self-Healing Polymers and Composites," Rev. Mater. Res., vol. 40, no. 179, 2010.

[4] C. Jeffrey, N. Dumas, A. Richardson, R. Bunyan, H. Mathias and P. Nouet, "Sensor Testing Through Bias Superposition.," Sensors and Actuators A: Physical, vol. 136, no. 1, pp. 441-455, 2007.

[5] N. Dumas, Z. Xu, K. Georgopoulos, R. J. T. Bunyan and A. Richardson, "A Novel Approach for Online Sensor Testing Based on an Encoded Test Stimulus," in 12th IEEE European Test Symposium, Freiburg, 2007.

[6] E. Sharif, A. Dorey and A. Richardson, "An integrated diagnostic reconfiguration (IDR) technique for fault tolerant mixed signal microsystem," in IEEE International Conference on Electronics, Circuits and Systems. Surfing the Waves of Science and Technology, Lisbon, 1998.

[7] B. Kaminska and K. Arabi, "Oscillation-test strategy for analog and mixed-signal integrated circuits," in Proceedings of 14th VLSI Test Symposium, 1996.

[8] H. Khan, Richardson, A and Q. Al-Gayem, "A Housekeeping Prognostic Health Management Framework for Microfluidic Systems," IEEE Transactions on Device and Materials Reliability, 13 April 2017.

[9] I. B. Obot, B. Ikenna and B. Onyeachu, "Electrochemical frequency modulation (EFM) technique: Theory and recent practical applications in corrosion research.," Journal of Molecular Liquids., vol. 249, pp. 83-86, 2017.

[10] C. A. Loto, "Electrochemical noise measurement technique in corrosion research," EleInt J. Elechtrochem. Sci., vol. 7, pp. 9248$9270,2012$.

[11] D. Cheneler, J. Bowen and G. Kaklamani, "Transient bioimpedance monitoring of mechanotransduction in artificial tissue during indentation," Journal of Electrical Bioimpedance, vol. 5(1), pp. 55-73, 2014.

[12] S. Gevorgian, H. Berg, H. Jacobsson and T. Lewin, "Application notes-basic parameters of coplanar-strip waveguides on multilayer dielectric/semiconductor substrates, Part 1: high permittivity superstrates. IEEE microwave magazine,", IEEE Microwaves, vol. 4, no. 2 , pp. $60-70,2003$.

[13] S. \&. A. S. Gevorgian, "Analytic approximations for multilayer substrate coplanar-plate capacitors. In Microwave Symposium Digest,", IEEE MTT-S International (pp. 4-pp), 2005. 